\section{Journal of Mathematical Analysis and Modeling \\ jmam. sabapub.com}

ISSN 2709-5924
$\mathrm{J}$ Math Anal \& Model

(2021)2(3) : 77-87

doi:10.48185/jmam.v2i3.318

\title{
Effect of quarantine and vaccination in a pandemic situation: a mathematical modelling approach
}

\author{
D. S. A. Aashiqur Reza ${ }^{a, *}$, Md. Noman Billah ${ }^{a}$, Sharmin Sultana Shanta $^{a}$ \\ ${ }^{a}$ Mathematics Discipline, Khulna University, Khulna-9208, Bangladesh
}

• Received: 31 July 2021 • Accepted: 17 September 2021 • Published Online: 30 December 2021

\begin{abstract}
When a pandemic occurs, it can cost fatal damages to human life. Therefore, it is important to understand the dynamics of a global pandemic in order to find a way of prevention. This paper contains an empirical study regarding the dynamics of the current COVID-19 pandemic. We have formulated a dynamic model of COVID-19 pandemic by subdividing the total population into six different classes namely susceptible, asymptomatic, infected, recovered, quarantined, and vaccinated. The basic reproduction number corresponding to our model has been determined. Moreover, sensitivity analysis has been conducted to find the most important parameters which can be crucial in preventing the outbreak. Numerical simulations have been made to visualize the movement of population in different classes and specifically to see the effect of quarantine and vaccination processes. The findings from our model reveal that both vaccination and quarantine are important to curtail the spread of COVID-19 pandemic. The present study can be effective in public health sectors for minimizing the burden of any pandemic.
\end{abstract}

Keywords: Mathematical model, Basic reproduction number, COVID-19 pandemic, Quarantine, Vaccination.

2010 MSC: 92D30, 37M05, 34A34, 34H05.

\section{Introduction}

Infectious diseases are mainly caused by many pathogenic micro-organisms like bacteria, viruses, fungi, and parasites. These types of pathogens seem to find a host body for the availability of their food and grow quickly. The host body keeps fighting against them but once the system is compromised, the pathogen overwhelms the host body and causes infections [1]. Once the host body gets infected, it shows up different sorts of symptoms of several diseases. Fever, coughing, rashes, muscle pain, fatigue, and diarrhoeal disease are the most common indications of infectious diseases. Such diseases can also be transmitted from one human body to another. The most common ways of transmissions are: through contact, sprays, splashes, inhalation, and sharps injuries [2].

\footnotetext{
*Corresponding author: aashiq.reza007@gmail.com
} 
The most recent outbreak of COVID-19 (SARS-CoV-2) has taken nearly $4.6+$ million lives and due to this, nearly $223+$ million people around the globe got infected [3]. Therefore, understanding the dynamics of an infectious disease, such as COVID-19 pandemic, is very important as it may lead to find the solutions to defend the adverse situation. Mathematical modelling has been proven as an important tool to analyze the dynamics of infectious diseases. This paper aims to form a model through which the dynamics of COVID-19 pandemic can be explained.

To describe the situation of infectious diseases, researchers have already used several mathematical modelling approaches. A study estimates the clinical severity of COVID-19 pandemic based on the transmission process [4]. It is found that fractional derivative can be a powerful tool to define the transmission with a global nature [5] whereas fractalfractional-ABC derivatives can supply novel parameters to control a disease [6]. Moreover, Laplace Adomian decomposition is an alternative way to derive an approximate explanation for the global infectious diseases like COVID-19 [7]. A model evaluated the impact of dynamic isolation and screening program on the epidemic of COVID-19 spread [8]. A modeling study found that with complete lockdown, the spread of COVID-19 will disappear, otherwise the disease will persist [9]. Another study noted that non pharmaceutical interventions have impacts on COVID-19 [10]. A model described how isolation and quarantine of asymptomatic people can control the spread [11]. It has been revealed that protection, death, exposure, and cure rates have impacts on the total population involving immigration with time [12]. A model investigated the human to human transmission and revealed that restriction along with testing and quarantine can decrease infected class [13]. Universal face masks at moderate level could stop post-lockdown reappearance and avert the next wave of COVID-19 pandemic [14]. Media awareness can also help preventing the spread of COVID-19 [15]. Media coverage along with non pharmaceutical interventions applied at right timings can stop the outbreak of COVID-19 [16] whereas delay in diagnosis can not eliminate the disease [17]. Environment can also work as a reservoir of COVID-19 and is able to spread the virus [18]. Models on vaccination show that vaccination can reduce infections $[19,20,21]$. Moreover, vaccinating a group with a certain priority will effectively eliminate the infectious diseases [22].

In this research, we aim to study the impact of quarantine and vaccination. The main goal of this study is to understand how vaccination coverage as well as quarantine process can be effective to control the COVID-19 pandemic. The paper is organized as follows: in Section 2, we present the formulation of our model. Section 3 represents the findings of our study. In Section 4, a brief discussion regarding the simulations of our model has been given and in Section 5, the important conclusions of our research have been highlighted.

\section{Materials and methods}

\subsection{Mathematical model}

Our model is conceptually a modified form of the traditional SIR model [23]. The interactions between the compartments are based on some assumptions. We have divided the population into six possible classes: susceptible $(S)$, asymptomatic $(A)$, infected $(I)$, recovered $(R)$, quarantined $(Q)$, and vaccinated $(V)$. We consider that susceptible population become infectious by coming contact with asymptomatic and infected individuals. Again, 
we have assumed that susceptibles are attaining self-quarantine or being quarantined by the rules imposed on them and asymptomatic population are also being quarantined. Recovery comes from both asymptomatic and infected compartments. The vaccination campaign is conducted only with the susceptibles. Recovered class can lose their immunity and vaccinated class can become asymptomatic due to the failure of vaccines. Based on all these assumptions, we can formulate our model (Figure 1) as follows:

$$
\left\{\begin{aligned}
\dot{S} & =\left(-\beta A-\alpha_{1} \beta I-\omega-\theta_{2}\right) S+\theta_{1} Q+\gamma R \\
\dot{A} & =\left(-\lambda_{2}-q_{1}+\beta S-\alpha_{2}\right) A+\alpha_{1} \beta S I+\sigma V+q_{2} Q \\
\dot{I} & =-\left(\lambda_{1}+\mu\right) I+\theta Q+\alpha_{2} A \\
\dot{R} & =-\gamma R+\lambda_{1} I+\lambda_{2} A \\
\dot{Q} & =\left(-\theta_{1}-\theta-q_{2}\right) Q+q_{1} A+\theta_{2} S \\
\dot{V} & =\omega S-\sigma V
\end{aligned}\right.
$$

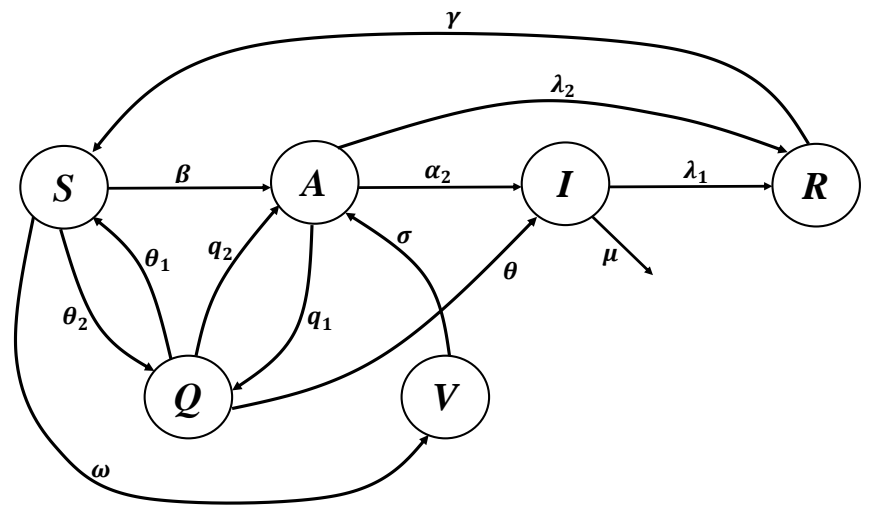

Figure 1: Diagram of model (2.1)

The term $\beta S A$ indicates that susceptibles are becoming asymptomatic when they interact or come contact with the asymptomatic individuals. Asymptomatic population recover after a certain period at a rate $\lambda_{2}$ or they may get infected by the disease after an incubation period with a rate $\alpha_{2}$. Some of the infected individuals are facing death as an impact of the disease at a rate $\mu$. Infected population are getting recovered with $\lambda_{1}$ rate. As we have considered the decline of immunity in our assumptions, some recovered population are going back to the susceptible class at a rate $\gamma$. Susceptibles are attaining self-quarantine or they are being quarantined due to some rules imposed on them. The rate at which susceptibles are being quarantined is $\theta_{2}$. Asymptomatic population are also being quarantined at a rate $q_{1}$. Quarantined population can also rejoin the susceptible class and asymptomatic class with rates $\theta_{1}$ and $\mathrm{q}_{2}$ respectively. Another portion from quarantined class can show some symptoms of the disease and get infected at a rate $\theta$. Susceptibles are getting vaccinated at a rate $\omega$. Vaccinated individuals can also become asymptomatic at a rate $\sigma$. Since we have considered a short time scale, we have neglected natural birth and death rates in our model. 


\subsubsection{Basic reproduction number for model (2.1)}

It is important to determine the basic reproduction number $\left(R_{0}\right)$ of a model. It can be interpreted as the number of secondary infections resulting from a single primary infection [24]. When $R_{0}>1$, it is an indication that the infectious disease is going to sustain in the community. On the other hand, the disease will die out when $R_{0}<1$.

The next generation matrix method has been used to find the basic reproduction number. Firstly, we have considered two groups $\mathrm{G}_{1}$ : asymptomatic, infected, and quarantined; and secondly, $G_{2}$ : susceptible, recovered, and vaccinated. Therefore, the infected block can be explained as:

$$
M=\left(\begin{array}{ccc}
-\lambda_{2}-q_{1}+\beta S-\alpha_{2} & \alpha_{1} \beta S & q_{2} \\
\alpha_{2} & -\left(\lambda_{1}+\mu\right) & \theta \\
q_{1} & 0 & -\left(\theta_{1}+\theta+q_{2}\right)
\end{array}\right)
$$

$M$ can be decomposed in $\mathcal{F}$ and $\mathcal{V}$, where $M=\mathcal{F}-\mathcal{V}$. Hence, we get

$$
\mathcal{F}=\left(\begin{array}{ccc}
\beta S & \alpha_{1} \beta S & 0 \\
0 & 0 & 0 \\
0 & 0 & 0
\end{array}\right)
$$

and

$$
\nu=\left(\begin{array}{ccc}
\alpha_{2}+\lambda_{2}+q_{1} & 0 & -q_{2} \\
-\alpha_{2} & \lambda_{1}+\mu & -\theta \\
-q_{1} & 0 & \theta_{1}+\theta+q_{2}
\end{array}\right)
$$

We obtain $\mathrm{R}_{0}$ by determining the eigenvalue of the matrix $\mathcal{F} \mathcal{V}^{-1}$ as follows:

$$
R_{0}=\frac{S \beta c_{2}}{c_{1}+c_{2} c_{3}}+\frac{S \beta \alpha_{1} c_{5}}{c_{4}\left(c_{1}+c_{2} c_{3}\right)}
$$

where

$c_{1}=-q_{1} q_{2}, c_{2}=\theta+q_{2}+\theta_{1}, c_{3}=q_{1}+\alpha_{2}+\lambda_{2}, c_{4}=\mu+\lambda_{1}, c_{5}=\theta q_{1}+\theta \alpha_{2}+q_{2} \alpha_{2}+$ $\alpha_{2} \theta_{1}$. Using the parameter values given in Table 1 , the value of $R_{0}$ is 1.9 .

\subsubsection{Initial values}

For the numerical simulations of model (2.1), we set $S(0)=9003322$ [15], $A(0)=500$, $I(0)=30$ [15], $R(0)=2, Q(0)=200$, and $V(0)=0$. Therefore, the initial total population is $\mathrm{N}(0)=9004054$. Besides initial values of the state variables, a proper simulation also depends on the values of the parameters. The parameter values of our model have been given in Table 1.

\subsubsection{Sensitivity analysis for model (2.1)}

Sensitivity analysis provides information about the crucial parameters of a mathematical model [25]. This tool is highly appreciated in research areas where researchers are willing to simulate their model to show how the situation may behave based on different parameters. Sensitivity analysis of the parameters which are included in the equation of 
Table 1: Parameter values.

\begin{tabular}{llll}
\hline Parameter & Description & Value & Source \\
\hline$\beta$ & Transmission rate & $3.112 \mathrm{e}-8$ & {$[15]$} \\
$\alpha_{1}$ & Proportion at which susceptibles are getting infected & 0.16 & {$[11]$} \\
$\alpha_{2}$ & Rate at which asymptomatic population become infected & $1 / 10$ & {$[18]$ Incubation $(2-14$ days) } \\
$\lambda_{1}$ & Recovery rate of asymptomatic & $1 / 14$ & {$[8,17]$} \\
$\lambda_{2}$ & Recovery rate of infected & $1 / 14$ & {$[8,17]$} \\
$\gamma$ & Rate from recovered to susceptible (immunity losing rate) & 0.0003 & {$[12]$} \\
$\theta$ & Rate at which quarantined population are getting infected & $3.2084 \mathrm{e}-4$ & {$[13]$} \\
$\theta_{1}$ & Rate from quarantined to susceptible & $1 / 14$ & Assumed \\
$\theta_{2}$ & Rate from susceptible to quarantined & $2 / 1000$ & Assumed \\
$q_{1}$ & Rate from asymptomatic to quarantined & 0.001 & Assumed \\
$q_{2}$ & Rate from quarantined to asymptomatic & 0.005 & Assumed \\
$\sigma$ & Rate from vaccinated to asymptomatic (failure rate of vaccination) & 0.002 & Assumed \\
$\omega$ & Rate from susceptible to vaccinated (vaccination rate) & 0.0005 & Assumed \\
$\mu$ & Death rate due to disease & 0.0209 & Assumed \\
\hline
\end{tabular}

Table 2: Sensitivity analysis for model (2.1)

\begin{tabular}{ll}
\hline Parameter & Sensitivity index \\
\hline$\beta$ & +1 \\
$\mathrm{q}_{2}$ & $+3.84 \mathrm{e}-4$ \\
$\theta_{1}$ & $-4.15 \mathrm{e}-4$ \\
$\theta$ & $+3.07 \mathrm{e}-5$ \\
$\alpha_{2}$ & -0.42 \\
$\lambda_{2}$ & -0.41 \\
$\mathrm{q}_{1}$ & -0.03 \\
$\alpha_{1}$ & +0.15 \\
$\mu$ & -0.33 \\
$\lambda_{1}$ & -0.12 \\
\hline
\end{tabular}

basic reproduction number $R_{0}$ has been conducted. It is expected that the sensitivity indices should vary from -1 to +1 . To find the sensitivity index of any parameter $p$, we have used the formula $\frac{\partial R_{0}}{\partial p} \frac{p}{R_{0}}$. After doing all the necessary calculations, we obtain the sensitivity of the parameters which has been given in Table 2 . From sensitivity analysis, it is clear that the most sensitive parameter is the transmission rate $\beta$ since it is with highest positive value.

\subsection{Mathematical model without vaccinated class}

In this subsection, we aim to see the effects of quarantine policy in absence of vaccination. Therefore, we have excluded the vaccinated compartment from model (2.1). Finally, the modified form of model (2.1) is as follows:

$$
\left\{\begin{aligned}
\dot{S} & =\left(-\beta A-\alpha_{1} \beta I-\theta_{2}\right) S+\theta_{1} Q+\gamma R \\
\dot{A} & =\left(-\lambda_{2}-q_{1}+\beta S-\alpha_{2}\right) A+\alpha_{1} \beta S I+q_{2} Q \\
\dot{I} & =-\left(\lambda_{1}+\mu\right) I+\theta Q+\alpha_{2} A \\
\dot{R} & =-\gamma R+\lambda_{1} I+\lambda_{2} A \\
\dot{Q} & =\left(-\theta_{1}-\theta-q_{2}\right) Q+q_{1} A+\theta_{2} S
\end{aligned}\right.
$$




\subsubsection{Basic reproduction number for model (2.2)}

For model (2.2), we denote the corresponding basic reproduction number by $\mathrm{R}_{0}^{\mathrm{q}}$. Since the dynamic patterns of $A, I$ and $Q$ are same both in models (2.1) and (2.2), the infected blocks for both models will be similar which suggests that $R_{0}^{q}$ will be equal to $R_{0}$. That means, $\mathrm{R}_{0}^{\mathrm{q}}=1.9$.

\subsubsection{Sensitivity analysis for model (2.2)}

Since $R_{0}^{q}=R_{0}$, the parameters that are involved in model (2.2) must have the same sensitivity indices as we have seen for model (2.1). Therefore, it is easy to demonstrate that for model (2.2), transmission rate $\beta$ is responsible for the spread of the disease since it has the highest positive sensitivity index (Table 2). Besides $\beta$, the quarantine related parameters with positive sensitivity indices are $q_{2}$ and $\theta$ whereas the parameters with negative indices (related to quarantine) are $\theta_{1}$ and $q_{1}$. Therefore, in order to control the COVID-19 pandemic in absence of vaccination process, the rates $\beta, q_{2}$ and $\theta$ need to be decreased and the rates $\theta_{1}$ and $q_{1}$ should be increased.

\subsection{Mathematical model without quarantined class}

This subsection deals with a modified form of model (2.1) by excluding the quarantined class. We aim to see the disease behaviour in absence of quarantine policy. Thus, the new model takes the following formation:

$$
\left\{\begin{aligned}
\dot{S} & =\left(-\beta A-\alpha_{1} \beta I-\omega\right) S+\gamma R \\
\dot{A} & =\left(-\lambda_{2}+\beta S-\alpha_{2}\right) A+\alpha_{1} \beta S I+\sigma V \\
\dot{I} & =-\left(\lambda_{1}+\mu\right) I+\alpha_{2} A \\
\dot{R} & =-\gamma R+\lambda_{1} I+\lambda_{2} A \\
\dot{V} & =\omega S-\sigma V
\end{aligned}\right.
$$

2.3.1. Basic reproduction number for model (2.3)

Let the basic reproduction number for model (2.3) is $\mathrm{R}_{0}^{v}$. The corresponding infected block for model (2.3) is as follows:

$$
M_{v}=\left(\begin{array}{cc}
-\lambda_{2}+\beta S-\alpha_{2} & \alpha_{1} \beta S \\
\alpha_{2} & -(\lambda+\mu)
\end{array}\right)
$$

Therefore,

$$
\mathcal{F}_{v}=\left(\begin{array}{cc}
\beta S & \alpha_{1} \beta S \\
0 & 0
\end{array}\right)
$$

and

$$
\mathcal{V}_{v}=\left(\begin{array}{cc}
\lambda_{2}+\alpha_{2} & 0 \\
-\alpha_{2} & \lambda_{1}+\mu
\end{array}\right)
$$

Finally,

$$
R_{0}^{v}=\frac{\beta S}{\alpha_{2}+\lambda_{2}}+\frac{\beta S \alpha_{1} \alpha_{2}}{\left(\alpha_{2}+\lambda_{2}\right)\left(\mu+\lambda_{1}\right)}
$$

Using the values of the parameters from Table 1 , we obtain $R_{0}^{v}=1.92$. 
Table 3: Sensitivity analysis for model (2.3)

\begin{tabular}{ll}
\hline Parameter & Sensitivity index \\
\hline$\beta$ & +1 \\
$\alpha_{2}$ & -0.44 \\
$\lambda_{2}$ & -0.42 \\
$\alpha_{1}$ & +0.15 \\
$\mu$ & -0.03 \\
$\lambda_{1}$ & -0.12 \\
\hline
\end{tabular}

\subsubsection{Sensitivity analysis for model (2.3)}

The sensitivity of $R_{0}^{v}$ has been performed and the sensitivity indices have been given in Table 3 from where we observe that the parameters $\beta$ and $\alpha_{1}$ are with positive sensitivity values (need to decrease) and the parameters $\alpha_{2}, \lambda_{1}$ and $\lambda_{2}$ are with negative sensitivity values (need to increase).

\section{Results}

(a)

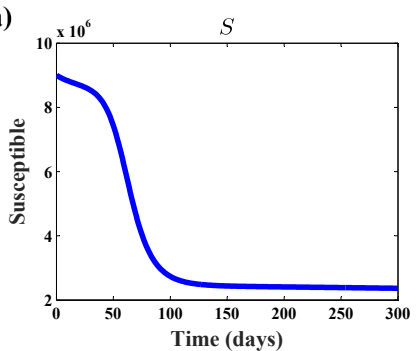

(d)

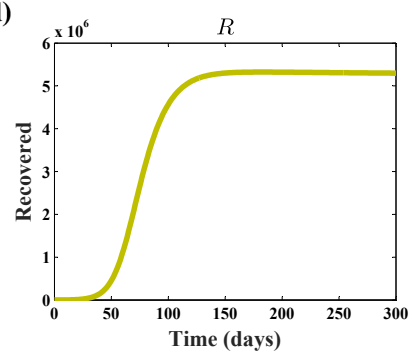

(b)

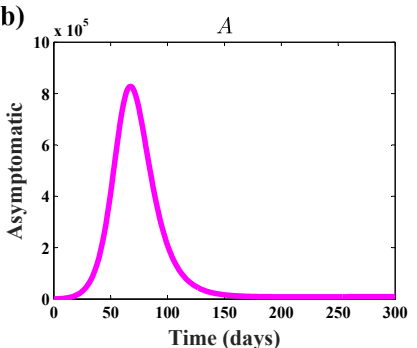

(e)

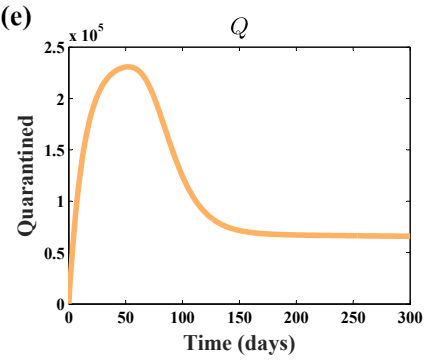

(c)
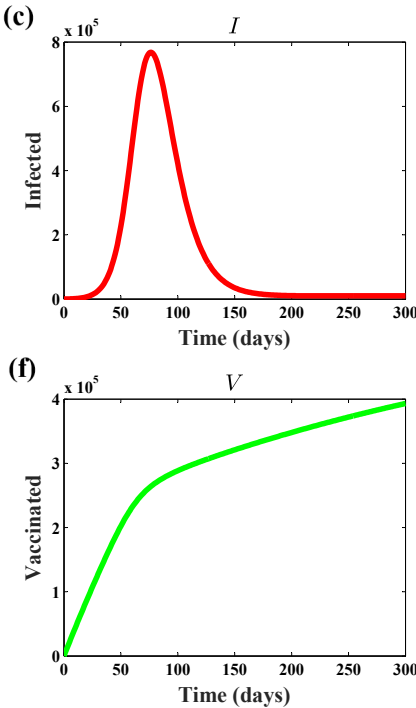

Figure 2: Simulation of model (2.1).

\subsection{Observations from model (2.1)}

Figure 2 represents the numerical simulation of model (2.1). Findings from this simulation confirm that importance should be given on both vaccination and quarantine processes. It is observed that if quarantine process continues along with vaccination, the progression of asymptomatic and infected classes will go nearly to zero after 150 days. For such scenario, $4 \%$ of the population will be vaccinated and nearly $0.7 \%$ of the population will be in quarantine at 300 days. Figure 3 shows the possible scenario regarding the infection. It is observed from Figure 3(a) that with the increase of transmission rate, 
(a)

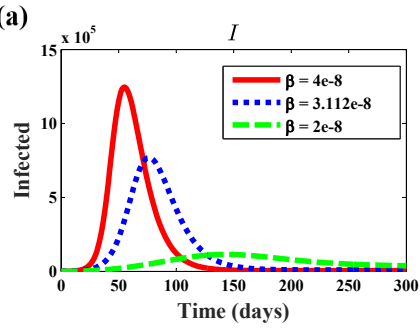

(b)

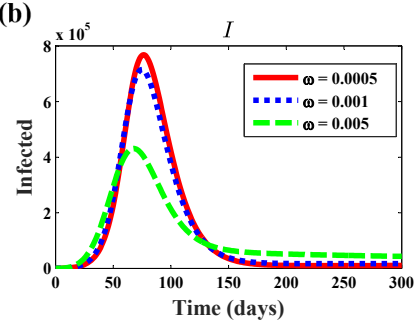

(c)

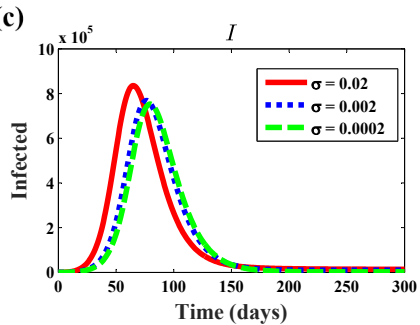

Figure 3: Simulation of model (2.1) representing infected class (a) as transmission rate varies, (b) as vaccination rate varies, (c) as failure rate of vaccination varies.

(a)

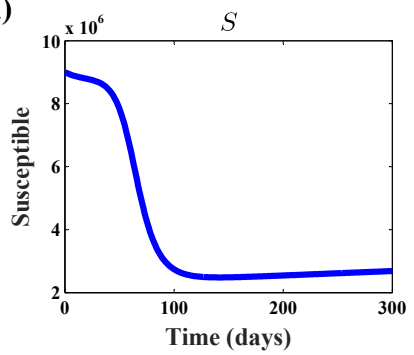

(d)

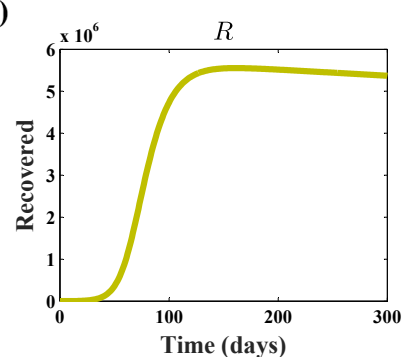

(b)

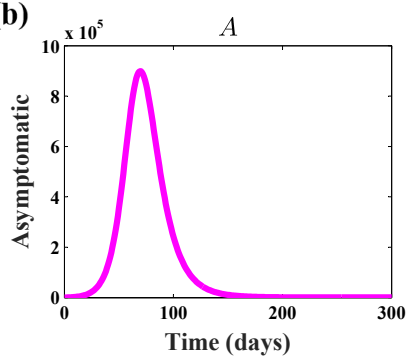

(e)

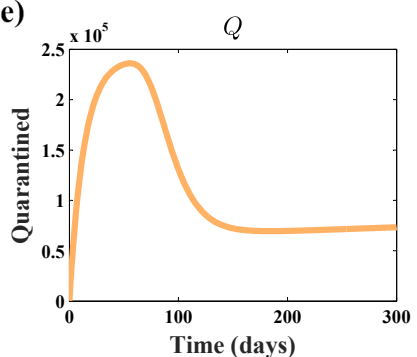

(c)

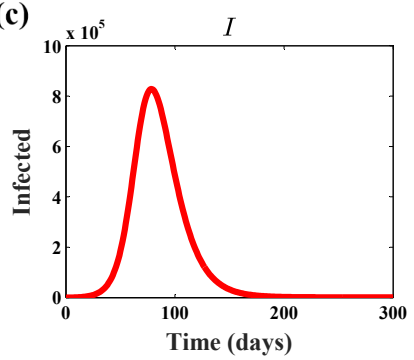

Figure 4: Simulation of model (2.2).

infected peak will increase and appear earlier. On the other hand, infected peak will decrease with an augmentation of vaccination rate (Figure 3(b)). From Figure 3(c), it is also understood that infection can be reduced with lower failure rate of vaccination.

\subsection{Observations from model (2.2)}

Simulation of model (2.2) shows that in absence of vaccination process, around 0.8 million people will become infected at the pandemic peak with 0.9 million asymptomatic cases (Figure 4). Moreover, susceptible curve is observed to take an upturn after 120 days which is an initial indication of the next wave of the pandemic.

\subsection{Observations from model (2.3)}

From Figure 5, we see the results obtained by simulating the model (2.3). If there is no quarantine policy, then according to the simulation, around 1 million population will be asymptomatic and nearly 0.8 million population will be infected at the peak of 
(a)

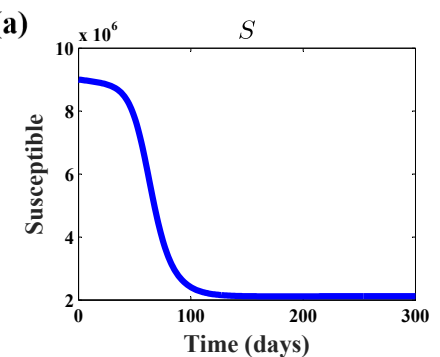

(d)

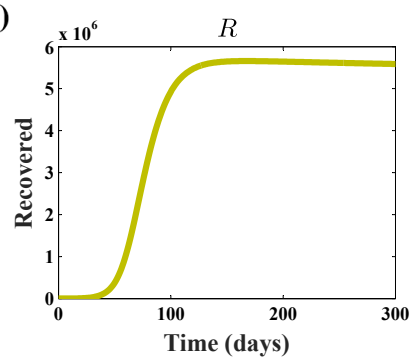

(b)

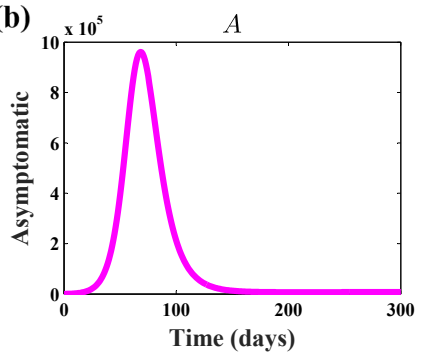

(e)

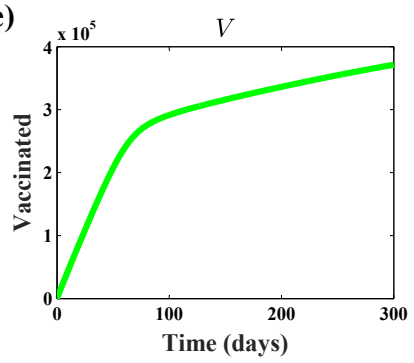

(c)

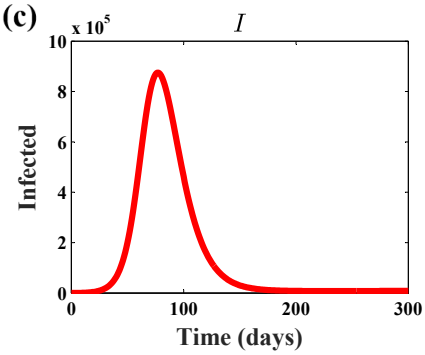

Figure 5: Simulation of model (2.3).

pandemic. In order to force the progression at low level, nearly $4 \%$ of the population should be vaccinated within 300 days.

\section{Discussion}

In this study, an analysis has been conducted based on the traditional SIR type model with extra compartments namely vaccination and quarantine processes. The total population have been divided into six different classes. The main goal of this research is to describe the importance of vaccination and quarantine processes to control the spread of COVID-19 pandemic. We have determined the basic reproduction number which is greater than 1. Moreover, sensitivity analysis gives the idea about the control parameters of our model. From sensitivity analysis, it is understood that transmission parameter plays a crucial role for spreading the virus. Therefore, to study any epidemic or pandemic situation, care should be given on the transmission rate. If measures can be taken to slow down the transmission rate, it will be possible to control the spread of the disease. Even a small decline of the transmission rate brings a reasonable change in the number of infected population. According to our model, both quarantine and vaccination are important during a disease period. In absence of vaccination, quarantine process can be a tool to curb the progression of the pandemic whereas the coverage of vaccines can control the spread of the virus. Infection will drop down to the low level with higher vaccination rate. However, infection is about to increase due to the failure rate of vaccination. This observation draws attention to assure the quality and effectiveness of the vaccines to control the pandemic. 


\section{Conclusions}

In this study, a general model has been proposed to study the dynamics of COVID-19 pandemic and to show the impact of quarantine and vaccination in controlling the spread of the disease. The model shows that the spread of the pandemic can be controlled by vaccinating around $4 \%$ of the total population over ten months period. Besides vaccination, quarantine is an option to curtail the pandemic. Comparisons have been made to investigate the outcomes of the proposed model by subdividing the model into two new models so that the effects of vaccination in absence of quarantine and the effects of quarantine in absence of vaccination can easily be identified. Such two models revealed that both quarantine and vaccination play important roles in reducing the community transmission of COVID-19 pandemic. Finally, the present model has successfully highlighted the impacts of the possible factors to bring up control over the pandemic situation. The model emphasizes on the facts of quarantine and vaccination processes to minimize the disease burden and possibly shows a path to eradicate the disease from communities. Attention should be drawn both on quarantine and vaccination processes because it is challenging to vaccinate the entire susceptible class within a very short period. A successful vaccination campaign is a time consuming process. This process includes the production of a vaccine, usability, and analysis of the possible side effects. The willingness of individuals to get vaccinated is another issue at the beginning of the vaccination campaign. To control the spread of the disease, quarantine is an effective way besides vaccination. When a proper vaccination campaign starts to take place, quarantine process should also be going on to control the spread. Thus, in the current COVID-19 situation, our present model could be informative to draw reasonable conclusions.

Author contributions: Conceptualization, D.A.R., M.N.B. \& S.S.S.; Methodology, Software, Validation \& Formal Analysis, D.A.R. \& S.S.S.; Investigation, S.S.S.; Writing-Original Draft Preparation, D.A.R. \& M.N.B.; Writing-Review \& Editing, S.S.S.; Supervision, S.S.S.

\section{References}

[1] Kotra LP (2007). Infectious Diseases. xPharm: The Comprehensive Pharmacology Reference, 1-2. https : //doi.org/10.1016/B978-008055232-3.60849-9

[2] How Infections Spread, Centers for Disease Control and Prevention, Available from: https://www .cdc. gov/infectioncontrol/spread/index.html

[3] Coronavirus, Worldometer, Available from: https://www.worldometers.info/coronavirus/

[4] Wu JT, Leung K, Bushman M, Kishore N, Niehus R, de Salazar PM, Cowling BJ, Lipstich M and Leung GM (2020). Estimating clinical severity of COVID-19 from the transmission dynamics in Wuhan, China. Nature Medicine 26 (4): 506-510 https://doi.org/10.1038/s41591-020-0822-7

[5] Redhwan SS, Abdo MS, Shah K, Abdeljawad T, Dawood S, Abdo HA and Shaikh SL (2020). Mathematical modeling for the outbreak of the coronavirus (COVID-19) under fractional nonlocal operator. Results in Physics 19: Article number 103610. https://doi.org/10.1016/j.rinp.2020.103610

[6] Almalahi MA, Panchal SK, Shatanawi W, Abdo MS, Shah K and Abodayeh K (2021). Analytical study of transmission dynamics of 2019-nCoV pandemic via fractal fractional operator. Results in Physics 24 : Article number 104045. https://doi.org/10.1016/j.rinp.2021.104045

[7] Thabet ST, Abdo MS and Shah K (2021). Theoretical and numerical analysis for transmission dynamics of COVID-19 mathematical model involving Caputo-Fabrizio derivative. Advances in Difference Equations 6: 1-17. https://doi.org/10.1186/s13662-021-03316-w 
[8] Islam MAI, Shanta SS and Rahman A (2021). Modeling SARS-CoV-2 spread with dynamic isolation. Mathematics in Applied Sciences and Engineering, Online First. https://doi .org/10.5206/mase/13886

[9] Bhadauria AS, Pathak R, Chaudhary M (2021). textitA SIQ mathematical model on COVID-19 investigating the lockdown effect. Infectious Disease Modelling 6: 244-257. https://doi.org/10.1016/j . idm. 2020.12.010

[10] Ngonghala CN, Iboi E, Eikenberry S, Scotch M, MacIntyre CR, Bonds MH and Gumel AB (2020). Mathematical assessment of the impact of non-pharmaceutical interventions on curtailing the 2019 novel Coronavirus. Mathematical Biosciences 325: Article number 108364. https://doi.org/10.1016/j.mbs. 2020.108364

[11] Serhani M and Labbardi H (2021). Mathematical modeling of COVID-19 spreading with asymptomatic infected and interacting peoples. Journal of Applied Mathematics and Computing 66 (1): 1-20. https: //doi.org/10.1007/s12190-020-01421-9

[12] Din RU, Shah K, Ahmad I and Abdeljawad T (2020). Study of transmission dynamics of novel COVID-19 by using mathematical model. Advances in Difference Equations 323: 1-13. https://doi .org/10.1186/ s13662-020-02783-x

[13] Ahmed I, Modu GU, Yusuf A, Kumam P and Yusuf I (2021). A mathematical model of Coronavirus Disease (COVID-19) containing asymptomatic and symptomatic classes. Results in Physics 21: Article number 103776. https://doi.org/10.1016/j.rinp.2020.103776

[14] Ngonghala CN, Iboi EA and Gumel AB. (2020). Could masks curtail the post-lockdown resurgence of COVID-19 in the US?. Mathematical Biosciences 329: Article number 108452. https://doi.org/10. $1016 / j$.mbs . 2020.108452

[15] Shanta SS and Biswas MHA (2020). The Impact of Media Awareness in Controlling the Spread of Infectious Diseases in Terms of SIR Model. Mathematical Modelling of Engineering Problems 7 (3): 368-376. https: //doi.org/10.18280/mmep.070306

[16] Li A, Wang Y, Cong P and Zou X. (2021). Re-examination of the impact of some non-pharmaceutical interventions and media coverage on the COVID-19 outbreak in Wuhan. Infectious Disease Modelling 6: 975-987. https://doi.org/10.1016/j.idm.2021.07.001

[17] Rong X, Yang L, Chu H and Fan M (2020). Effect of delay in diagnosis on transmission of COVID-19. Mathematical Biosciences and Engineering 17 (3): 2725-2740. https://doi.org/https://doi.org/ $10.3934 / \mathrm{mbe} .2020149$

[18] Yang C and Wang J (2020). A mathematical model for the novel coronavirus epidemic in Wuhan, China. Mathematical Biosciences and Engineering 17 (3): Article number 2708. https://doi.org/https: //doi.org/10.3934/mbe. 2020148

[19] Ramos AM, Vela-P'erez M, Ferr'andez MR, Kubik AB and Ivorra B (2021). Modeling the impact of SARS-CoV-2 variants and vaccines on the spread of COVID-19. Communications in Nonlinear Science and Numerical Simulation 102: Article number 105937. https://doi.org/10.1016/j.cnsns. 2021. 105937

[20] Kumar P, Erturk VS and Murillo-Arcila M (2021). A new fractional mathematical modelling of COVID-19 with the availability of vaccine. Results in Physics 24: Article number 104213. https://doi.org/10. $1016 / j$.rinp. 2021.104213

[21] Ferranna M, Cadarette D and Bloom DE (2021). COVID-19 Vaccine Allocation: Modeling Health Outcomes and Equity Implications of Alternative Strategies. Engineering 7 (7): 924-935. https://doi.org/10. $1016 / j$.eng. 2021.03 .014

[22] Rahman SMA and Zou X (2015). Modelling the impact of vaccination on infectious diseases dynamics. Journal of Biological Dynamics 9 (Supplement 1): 307-320. https://doi.org/10.1080/17513758. 2014.986545

[23] Kermack WO and McKendrick AG (1927). A contribution to the mathematical theory of epidemics. Proceedings of the Royal Society A 115 (772): 700-721. https://doi.org/10.1098/rspa.1927.0118

[24] Van den Driessche P and Watmough J (2002). Reproduction numbers and sub-threshold endemic equilibria for compartmental models of disease transmission. Mathematical Biosciences 180 (1-2): 29-48. https: //doi.org/https://doi.org/10.1016/S0025-5564(02)00108-6

[25] Chitnis N, Hyman JM and Cushing JM (2008). Determining important parameters in the spread of malaria through the sensitivity analysis of a mathematical model. Bulletin of Mathematical Biology 70 (5): Article number 1272. https://doi.org/https://doi.org/10.1007/s11538-008-9299-0 